\title{
On roughness-induced adhesion enhancement
}

\author{
M. Ciavarella \\ Politecnico di BARI. Center of Excellence in Computational Mechanics. Viale Gentile \\ 182,70126 Bari.Mciava@poliba.it
}

\begin{abstract}
While adhesion reduction due to roughness is not surprising, roughness induced adhesion remained a puzzle until recently Guduru and coworkers have shown a very convincing mechanism to explain both the increase of strength and of toughness in a sphere with concentric single scale of waviness. Kesari and coworkers have later shown some very elegant convenient asymptotic expansion of Guduru's solution. This enhancement is very high and indeed, using Kesari's solution, it is here shown to depend uniquely on a Johnson parameter for adhesion of a sinusoidal contact. However, counterintuitively, it leads to unbounded enhancement for conditions of large roughness for which Johnson parameter is very low. Guduru postulated that this enhancement should occur after sufficiently large pressure has been applied to any spherical contact. Also, that although the enhancement is limited to the JKR regime of large soft materials with high adhesion, the DMT limit for the smooth sphere is found otherwise. However, for hard materials, even the DMT limit for the smooth solids is very hard to observe, which suggest that also adhesion reduction is yet not well understood.

The limitations of the assumption of simply connected area is here further discussed, and a well known model for hard particles in contact with rough planes due to Rumpf is used to show that in the range where unbounded increase is predicted, orders of magnitude reduction is instead expected for rigid solids. We suggest that Guduru's model may be close to an upper bound to adhesion of rough bodies, while the Rumpf-Rabinovich model may be close to a lower bound.
\end{abstract}

Keywords:

Roughness, Adhesion, Guduru's theory, Fuller and Tabor's theory 


\section{Introduction}

Fuller and Tabor (1975) were perhaps the first to measure adhesion of low modulus materials like smooth rubber lenses against roughened surfaces, and clearly showed very small amounts of roughness amplitude (few microns) were sufficient to destroy adhesion almost completely. They then proceeded to develop an asperity model, which is commonly believed not to permit enhancement of adhesion, but instead very rapid extinction 1 . In nature, various mechanisms have been shown to lead to adhesion enhancements in insects which use adhesion for their locomotion including varying the shape of each contact as well as increasing the number of them, so as to obtain a benefit from contact splitting (Hui et al., 2004, Kamperman et al, 2010, Gao \& Yao, 2004, Yao \& Gao, 2006). Adhesion enhancement was measured by Briggs \& Briscoe (1977), and Fuller \& Roberts (1981), who were unable to explain the data, particularly the increase of energy dissipation. Persson (2002) postulated that an increase of adhesion may occur for the increase of surface area induced by roughness, but later on this has been shown to be not the reason for the enhancement clearly explained by Guduru and collaborators (Guduru (2007), Guduru \& Bull (2007), Waters et al (2009)). Guduru's solution is a very classical contact mechanics exact solution assuming a simply connected contact area develops in a spherical contact having a concentric axisymmetric waviness. Clearly, as it was apparent also to Guduru (2007), there are some limitations for this solution to hold, as one expects the contact to occur only on the crests of the waviness, for "sufficiently" large amplitude roughness and this would limit the enhancement shown in the simply connected contact area model. The separated contact solution is unfortunately not possible in closed form, and therefore it was not studied by Guduru, who noticed however the is a large set of conditions for which we could assume it holds. Guduru's theory is very brilliantly described and corroborated by experiments, and serves the main purpose of showing when the enhancement can occur. However, we move here from a opposite perspective, trying to understand why the large enhancement is not commonly observed. We will therefore discuss two main aspects of the solution: the assumption of simply

\footnotetext{
${ }^{1}$ In reality, reduction is assumed from the outset in the model, as Fuller and Tabor postulated that the smooth sphere case corresponded to the aligned asperity case in the nominally plane model.
} 
connected contact area, and the assumption of JKR regime.

Regarding the first aspect, Guduru (2007) writes a condition on the monotonicity of the profile function which guarantees a simply connected area, but limits the amplitude of roughness in fact to regions where the enhancement is not too large. Guduru recognized that the condition is over-restrictive, in that, for sufficiently large pressure during loading, we may expect the simply connected area to being established, and therefore full enhancement may occur. Regarding the second aspect, Waters et al (2009) develop a Maugis-Dugdale solution with an annulus of uniform tension at the edge of the contact area, showing enhancement is limited to the JKR regime. However, they seem to imply, at least with the limited set of parameters they study, the limit should be that of a DMT smooth sphere, which in the case of a rough sphere, is in fact, although not an enhancement, still very high. We will therefore consider a limit case, that of rigid bodies in contact, showing that the limit is more complex than that, and permitting a very large reduction indeed. Much of the debate on adhesion of spherical bodies has concentrated on the transition from the DMT theory (see Greewood, 2007 for a detailed treatment about this controversial theory) to the JKR limit. However, the two theories differ, for pull-off, only on a small prefactor $(3 / 2$ in JKR, and 2 in DMT), whereas the more we understand about the effect of roughness on adhesion, the more we are confused between orders of magnitude reduction (as it is commonly observed) and 1 order of magnitude enhancement (or higher?), as Guduru's theory shows. This note therefore attempts to compare various results in order to hopefully arrive at a better comprehension between these extreme limits.

In some of the developments, we use the recently developed asymptotic expansion of Guduru's solution developed by Kesari et al (2010,2011), finding some reduced parameter dependence in the solution, which in fact is exactly a JKR solution, different in the loading and the unloading phase. We examine therefore where this solution holds, therefore providing some hopefully insight in further understanding of the more general complex problem of adhesion in rough surfaces which, in view of the competition between enhancement and reduction mechanisms, is still not well understood.

\section{Guduru-Kesari theory}

Waters et al (2009) give a good summary of Guduru's theory and experiments. They have a surface defined as $f(r)=\frac{r^{2}}{2 R}+A\left(1-\cos \frac{2 \pi r}{\lambda}\right)$, where $R$ 
is the sphere radius, $\lambda$ is wavelength of roughness, $h$ is its amplitude, and $A$ can be both positive in the case of a central convex asperity, and negative, for a central concave trough. The solution of the contact problem with adhesion is possible assuming a simply connected contact area, as a function of two parameters

$$
\alpha_{G}=\frac{A R}{\lambda^{2}} \quad, \quad \beta_{G}=\frac{\lambda^{3} E^{*}}{2 \pi w R^{2}}
$$

where $w$ is the surface energy, and $E^{*}$ the plane strain elastic modulus. The results are given in terms of a dimensionless load $\widehat{P}=P /\left(\frac{3}{2} \pi w R\right)$ so that $\widehat{P}=1$ corresponds to the smooth sphere, and also for dimensionless approach $\widehat{h}=h R / \lambda^{2}$, and contact radius $\widehat{a}=a / \lambda$, as

$$
\begin{aligned}
& \widehat{P}_{G}(\widehat{a})=\frac{8}{3} \beta\left[\frac{2}{3} \widehat{a}^{3}+\alpha\left(\frac{4 \pi^{2} \widehat{a}^{3}}{3}+\frac{\pi \widehat{a}}{2} H_{1}(2 \pi \widehat{a})-\pi^{2} \widehat{a}^{2} H_{2}(2 \pi \widehat{a})\right)\right]-\frac{8}{3} \sqrt{\beta \widehat{a}^{3}} \\
& \widehat{h}_{G}(\widehat{a})=\widehat{a}^{2}+\alpha \pi^{2} \widehat{a} H_{0}(2 \pi \widehat{a})-\sqrt{\widehat{a} / \beta}
\end{aligned}
$$

Kesari et al $(2010,2011)$ have developed a very elegant "envelope" solution of the Guduru problem. The envelop is obtained by joining in an asymptotic solution for small roughness sizes, in particular $\lambda<<a$ : said otherwise, there much be enough wavelengths of roughness in the contact area - a condition quite close to what was used in the Archard cascade process of redistribution of loads in the adhesionless contact problem (Ciavarella et al. (2000)), and which can be checked a posteriori. They notice that the solution has some simplified behaviour but do not further discuss this aspect. The envelop solution in terms of load and indentation depth is

$$
\begin{aligned}
& P_{K}(a)=\frac{4}{3 R} E^{*} a^{3}-a^{3 / 2}\left(\sqrt{8 \pi w E^{*}} \pm 2 \pi E^{*} \frac{A}{\sqrt{\lambda}}\right) \\
& h_{K}(a)=\frac{a^{2}}{R}-a^{1 / 2}\left(\sqrt{\frac{2 \pi w}{E^{*}}} \pm \pi \frac{A}{\sqrt{\lambda}}\right)
\end{aligned}
$$

where we have grouped the term with the same power in contact radius $a-$ the theory obviously corresponds to the known JKR theory for $\frac{A}{\sqrt{\lambda}}=0$.

\subsection{Reformulation of Kesari equations}

An obvious remark about the equations (4,5) is that they are exactly those of the JKR theory also in the case of roughness, but with a corrected 
(enhanced or reduced, respectively for unloading or loading) surface energy

$$
\begin{aligned}
& P(a)=\frac{4}{3 R} E^{*} a^{3}-a^{3 / 2} \sqrt{8 \pi w E^{*}}\left(1 \pm \frac{1}{\sqrt{\pi} \alpha_{K L J}}\right) \\
& h(a)=\frac{a^{2}}{R}-a^{1 / 2} \sqrt{\frac{2 \pi w}{E^{*}}}\left(1 \pm \frac{1}{\sqrt{\pi} \alpha_{K L J}}\right)
\end{aligned}
$$

where

$$
\alpha_{K L J}=\sqrt{\frac{2 w \lambda}{\pi^{2} E^{*} A^{2}}}
$$

is the parameter Johnson (1995) introduced for the JKR adhesion of a nominally flat contact having a single scale sinusoidal waviness of amplitude $A$ and wavelength $\lambda$. We can also recast the JKR "envelope" equations of Kesari et al (2011) in terms of only one of the Guduru dimensionless parameters, $\beta_{G}$ together with $\alpha_{K L J}$

$$
\begin{aligned}
& \widehat{P}_{K}(\widehat{a})=\frac{8}{3} \beta_{G} \widehat{a}^{3}-4 \sqrt{\widehat{a}^{3} \beta_{G}}\left(1 \pm \frac{1}{\sqrt{\pi} \alpha_{K L J}}\right) \\
& \widehat{h}_{K}(\widehat{a})=\widehat{a}^{2}-\left(\frac{\widehat{a}}{\beta_{G}}\right)^{1 / 2}\left(1 \pm \frac{1}{\sqrt{\pi} \alpha_{K L J}}\right)
\end{aligned}
$$

It is therefore very interesting that the increase of adhesion should scale with the same parameter of sinusoidal contact. It is also very interesting and unexpected a priori that an important value for this parameter in eqt. (9, 10) is

$$
\alpha_{K L J}=\frac{1}{\sqrt{\pi}} \simeq 0.56
$$

which is exactly the value for which the sinusoid self-flattens to full contact under no applied load. In fact, the behaviour of a sinusoidal contact should be explained in few words, following Fig.1, where $\alpha_{K L J}$ is indicated as $\alpha_{0}$ because in general, for a multiscale roughness, this parameter can only be defined appropriately to a single sinusoid, and in particular tends to increase for low fractal dimensions, which is the common case observed in practice (see Afferrante et al, 2015). 


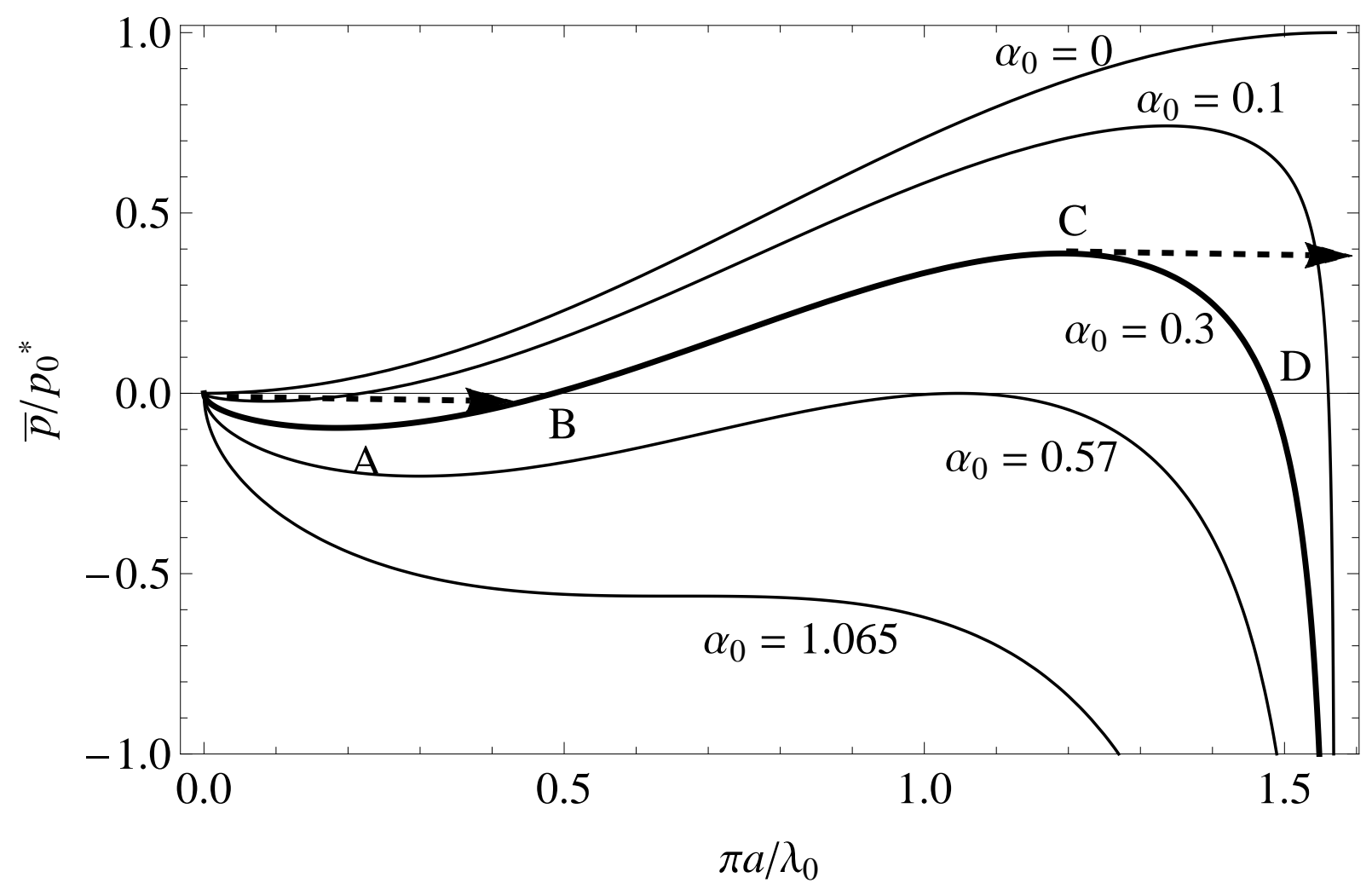

Fig.1. Behaviour of a nominally flat single sinusoidal contact

Suppose we follow the curve corresponding to $\alpha_{0}=0.3$. Under zero load, since the curve is decreasing (and hence unstable), the contact will "jump" to point B, similarly to any JKR solution. From this point on, a compressive load $\bar{p}$, moves the system along the curve $\mathrm{BC}$, until at point $\mathrm{C}$, again instability occurs and complete contact occurs. The maximum mean pressure needed to establish full contact is a fraction $\kappa$ of $p_{0}^{*}$, where $p_{0}^{*}=\pi E^{*} A / \lambda$ is the pressure for full contact without adhesion, and reduces with $\alpha_{0}$. For $\alpha_{0}>\alpha_{c r} \simeq 0.56$, partial contact does not occur and the surfaces immediately snap together until full contact occurs. At that point, when contact is established, it can be maintained also for negative arbitrarily high (tensile) mean pressures, provided $\bar{p} \geq \eta p_{0}^{*}$, where $\eta$ is a negative parameter, function of $\alpha_{0}$.

Notice there is a discussion in Waters et al (2009), based on qualitative energy balance, which essentially repeats the same process in Johnson's 
sinusoid $\alpha_{K L J}>0.56$, which for Guduru's parameter becomes gives

$$
\alpha_{G}<\frac{1}{\sqrt{2 \pi \beta_{G}}}
$$

Waters et al (2009) remark, correctly, that this condition only approximately estimates for which parameters the roughness is flattened and simply connected area is established spontaneously — that is, within the macroscopic contact area.

Returning to the actual Guduru model of a spherical contact with sinusoidal waviness, it wasn't necessary that the same parameter relative to the waviness in Guduru's solution appeared, and indeed, it did not appear in Guduru's solution, but only in its envelope from Kesari's solution. Assuming a simply connected area, the condition $\alpha_{K L J}>0.56$ seems sufficient to guarantee that this solution is appropriate, but as we shall see, enhancement is this region is relatively low, and instead, surprisingly, the largest enhancement occurs for low $\alpha_{K L J}$. We can estimate the actual "radius of spontaneous contact" therefore precisely from the full Guduru solution, and indeed also approximately from Kesari's solution.

It would seem that $\alpha_{K L J}>0.56$ shows an important transition too: the loading curve envelope becomes Hertzian for this value, and "less than Hertzian" for lower values $\alpha_{K L J}<0.56$, which in contrast would be the values where the enhancement of pull-off load would be even more than 4 . In particular, an effective energy on loading and unloading, respectively, can be defined as

$$
\sqrt{w_{\text {eff,loading }}}=w\left(1-\frac{1}{\sqrt{\pi} \alpha_{K L J}}\right) \quad ; \quad w_{\text {eff,unloading }}=w\left(1+\frac{1}{\sqrt{\pi} \alpha_{K L J}}\right)^{2}
$$

where we have left the square root for $w_{\text {eff,loading }}$ to take into account that for $\alpha_{K L J}<1 / \sqrt{\pi}, \sqrt{w_{\text {eff,loading }}}$ should be negative.

Notice that for the Kesari expansion to be valid, one needs $\widehat{a}>>1$. Therefore, for the condition at pull-off to be reasonably evaluated from this analysis, one needs in general low values of $\beta_{G}<<1$. In this limit only, one could use pull-off from the unloading curve, so the size of contact area at pull-off is

$$
\widehat{a}_{c, l o w \beta_{G}}^{3}=\frac{9 \pi}{4} \frac{R^{2}}{\lambda^{3}} \frac{w}{E^{*}}\left(1+\frac{1}{\sqrt{\pi} \alpha_{K L J}}\right)^{2}=\frac{9}{8 \beta_{G}}\left(1+\frac{1}{\sqrt{\pi} \alpha_{K L J}}\right)^{2}
$$


and the actual value of pull-off is

$$
\widehat{P}_{\min , \operatorname{low} \beta_{G}}=-\left(1+\frac{1}{\sqrt{\pi} \alpha_{K L J}}\right)^{2}
$$

Instead, the size of the contact area when the load is zero, is obtained from the loading curve (only for $\alpha_{K L J}>1 / \sqrt{\pi}$ ) as

$$
\widehat{a}_{e q, l o w \beta_{G}}^{3}=\frac{9}{2 \beta_{G}}\left(1-\frac{1}{\sqrt{\pi} \alpha_{K L J}}\right)^{2}
$$

and this suggests an alternative map from Fig.5 of Waters et al (2009) where dependence on $\alpha_{G}, \beta_{G}$ is shown. Indeed, in view of the convenience of writing Kesari's equation in terms of $\alpha_{K L J}$, perhaps a clearer notation is to rewrite the Guduru equations in terms of $\beta_{G}, \alpha_{K L J}$ instead of their original $\alpha_{G}, \beta_{G}$ using $\alpha_{G}=\frac{1}{\pi^{3 / 2} \alpha_{K L J} \sqrt{\beta_{G}}}$ and obtaining

$\widehat{P}_{G}(\widehat{a})=\frac{8}{3} \beta_{G} \widehat{a}^{3}+\left[\frac{4 \sqrt{\beta_{G}}}{\pi^{3 / 2} \alpha_{K L J}}\left(\frac{4 \pi^{2} \widehat{a}^{3}}{3}+\frac{\pi \widehat{a}}{2} H_{1}(2 \pi \widehat{a})-\pi^{2} \widehat{a}^{2} H_{2}(2 \pi \widehat{a})\right)\right]-4 \sqrt{\beta_{G} \widehat{a}^{3}}$

$\widehat{h}_{G}(\widehat{a})=\widehat{a}^{2}+\left[\frac{\pi^{1 / 2} \widehat{a} H_{0}(2 \pi \widehat{a})}{\alpha_{K L J} \sqrt{\beta_{G}}}\right]-\sqrt{\frac{\widehat{a}}{\beta_{G}}}$

where the terms under square parentheses cause the fluctuation in Guduru's equations, and are substituted in the envelop by the factors $\left(1 \pm \frac{\sqrt{\pi}}{\alpha_{K L J}}\right)$.

In fig.2,3 we show some examples of load-approach, approach-area and load-area, in 2 interesting cases with low $\beta_{G}$ : where Kesari's envelope (represented also here as blue and red curves) works well, and with low and with high $\alpha_{K L J}$. At low $\alpha_{K L J}$, the enhancement in pull-off is greater and the loading curves actually fold on each other in the load-approach diagram (Fig.1a). As the Kesari's equation predict, the spontaneous jump into contact practically does not exist, and therefore the large enhancement can only be obtained with sufficiently large pressure during the loading stage, and Kesari et al $(2010,2011)$ give also additional results on how to compute the loading and unloading curves, as well as the integral of the envelop curve to compute energy dissipation. 

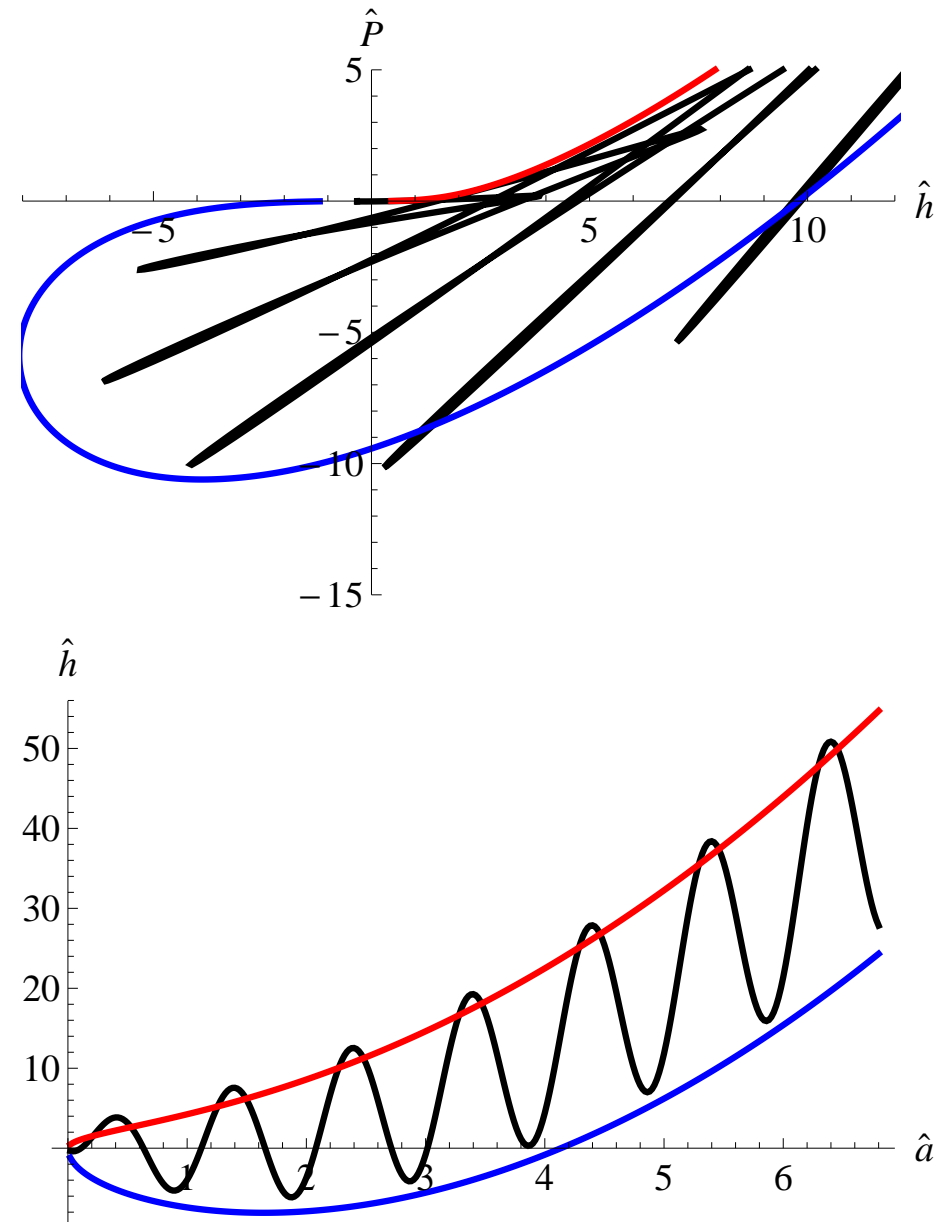

(a)

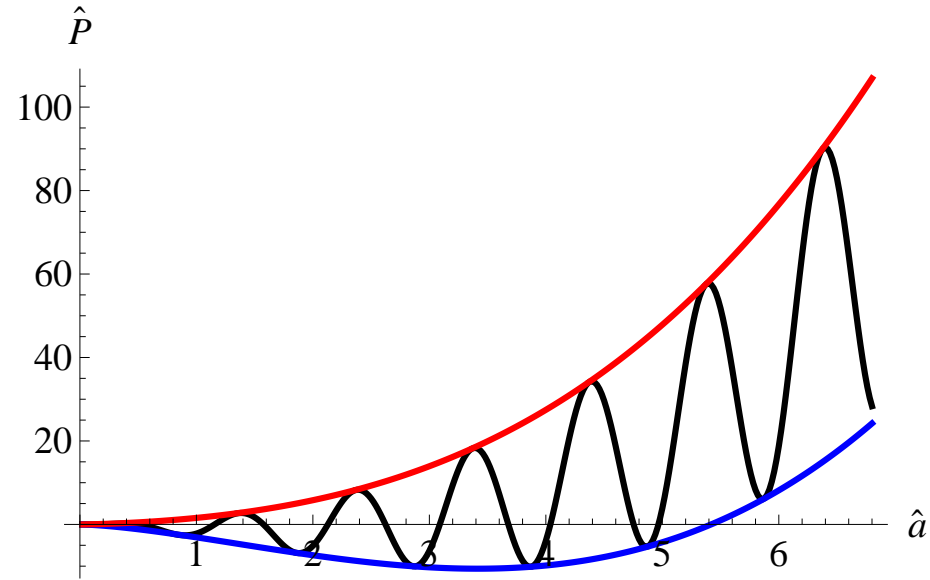

Fig.2 - Load-approach (a), approach-area (b) and load-area (c), for low 
$\alpha_{K L J}=0.25$ and low $\beta_{G}=0.15$. Blue and red lines are the Kesari envelopes for unloading and loading, respectively. Notice that in this case of $\alpha_{K L J}=0.25$ the curves on loading fold on each other. 


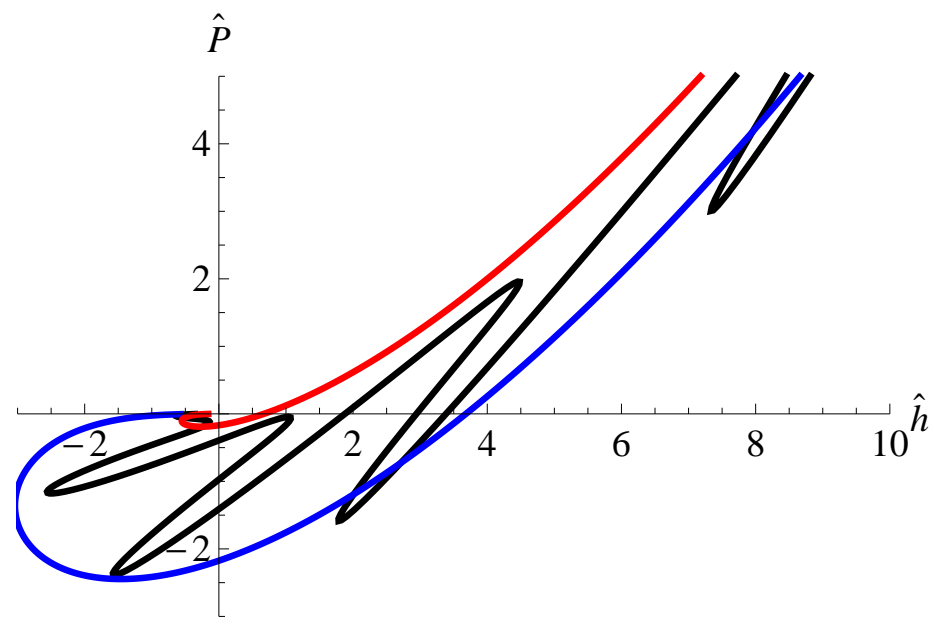

(a)
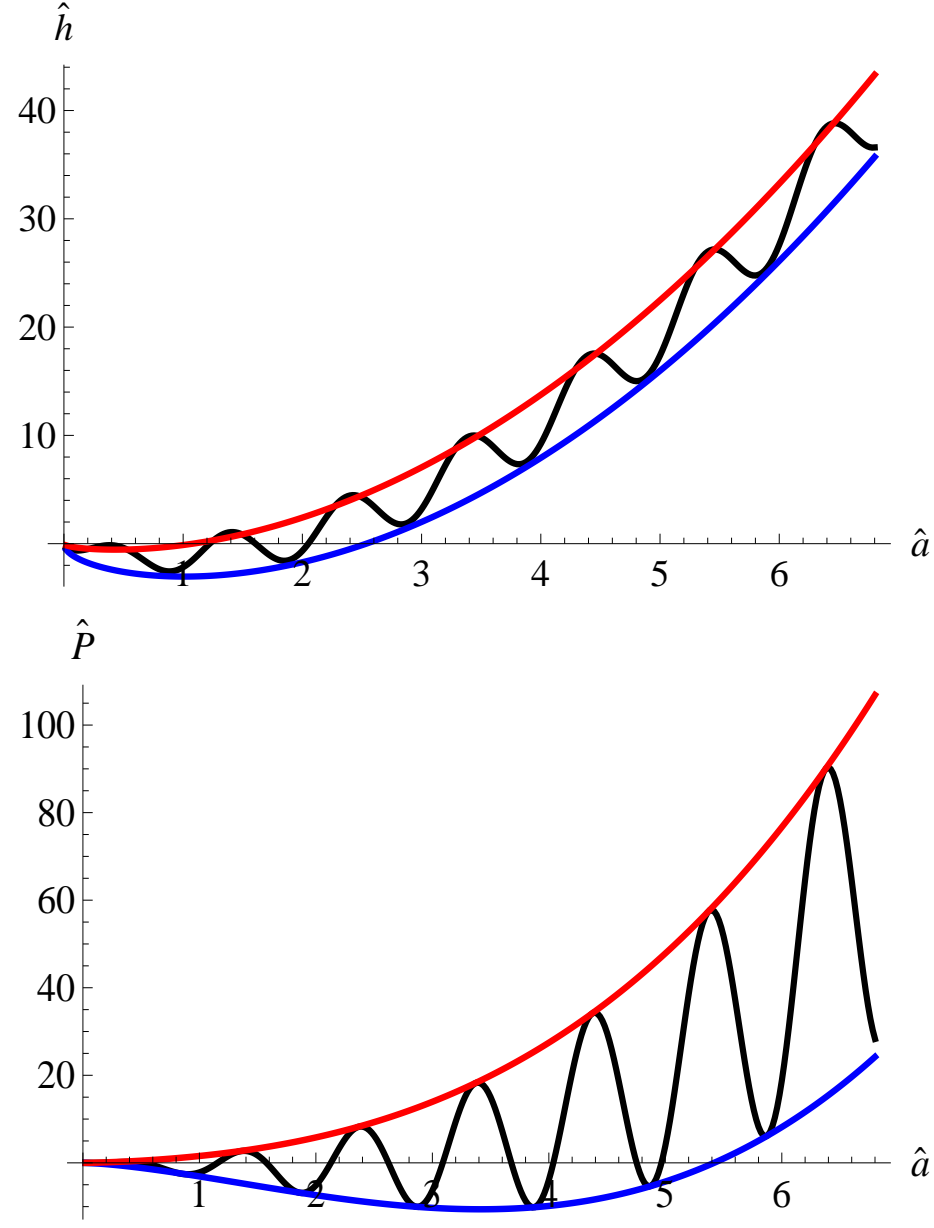
Fig.3 - Load-approach (a), approach-area (b) and load-area (c), for high $\alpha_{K L J}=1$ and low $\beta_{G}=0.15$.Blue and red lines are the Kesari envelopes for unloading and loading, respectively

For larger $\alpha_{K L J}$, instead, we have more noticeable spontaneous jump into contact and in this particular case, unloading from this point already seems to lead to a value of pull-off close to the Kesari envelope. Notice that Guduru (2007) had attempted an empirical fit for the pull-off enhancement of the type

$$
\widehat{P}_{\min }=-\left(1+\frac{C}{w^{\beta}}\right)
$$

where $\beta$ was observed to vary between 0.5 and 0.9 . The equation above (15) seems to justify this, considering that $\alpha_{K L J} \sim \sqrt{w}$, but does not permit a better fit including the deviations from Kesari's expansion at large $\beta_{G}$.

The enhancement of pull-off defined as $\left|\widehat{P}_{\min }\right|$ is shown in Fig.4 in the asymptotic limit at low $\beta_{G}$ i.e. from eqt.(15). It is clear that enhancements are small when $\alpha_{K L J}>10$ (about $10 \%$ increase, and less), significant in $\alpha_{K L J}<5$, has a value of 4 at the critical value $\alpha_{K L J}=0.56$ and continues to increase with lower $\alpha_{K L J}$. This is significant as the factor $\alpha_{K L J}$ measures the how sticky is the waviness in itself, and all the equations are saying is that a very sticky small amplitude waviness doesn't add nor destroy the stickiness of the macroscopic sphere. The biggest enhancement would seem to occur, surprisingly perhaps, at low values of $\alpha_{K L J}$, when the value of pull-off in dimensional terms would seem to be

$$
P_{\text {min }, \lim } \rightarrow-\frac{3}{2} \pi w R\left(\frac{1}{\sqrt{\pi} \alpha_{K L J}}\right)^{2}=-\frac{3}{4} \pi^{2} E^{*} R\left(\frac{A}{\sqrt{\lambda}}\right)^{2}
$$

which no longer depends on surface energy and seems instead to be related to elastic modulus alone and geometrical quantities2. This limit does not have

\footnotetext{
${ }^{2}$ Let us check this equation for example with atomic roughness, of both amplitude and wavelength $a_{0}$. As $\frac{w}{E^{*}}=l_{a}$, and for Lennard-Jones potential, $l_{a} / a_{0}=0.05$

$$
P_{\min , \lim } \rightarrow-\frac{3}{4} \pi^{2} E^{*} R a_{0}=-15 \pi^{2} w R=-148 w R
$$

which in fact corresponds to an enhancement with respect to the smooth sphere (?) of a factor $\frac{148}{1.5}=100$. But one could take wavelength of size $a_{0}$ and higher amplitude, and this would grow even further with $A^{2}$ without any apparent limit.
} 
much sense but it is interesting to remark that, while it is easy to understand why the uniform pressure shape of a contact could lead to theoretical strength in the contact (Gao \& Yao, 2004), although this limit is sensitive to shape at large sizes, here we seem to reach very high adhesion limits with a spherical contact, of very large size, adding roughness, which normally is instead considered to be a detrimental factor.

Clearly, there must be a limit when roughness separates the contact into multiply connected areas: in that region, pull-off enhancement may still be possible if a contact splitting effect prevails over the effect of competing elastic deformation of the asperities at different heights as distributed over the spherical shape.

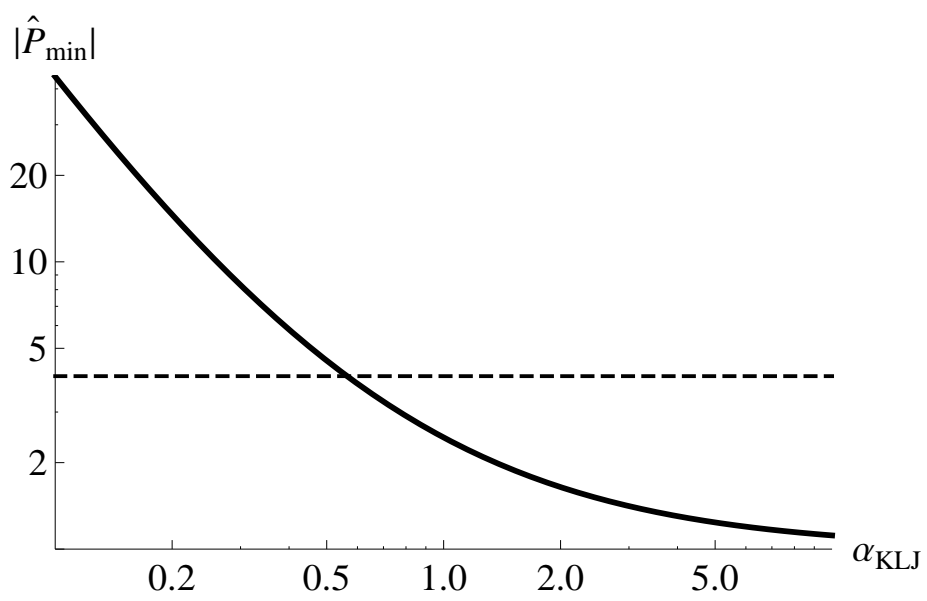

Fig.4 - Asymptotic dependence of pull-off enhancement on $\alpha_{K L J}=\sqrt{\frac{2 w \lambda}{\pi^{2} E^{*} A^{2}}}$ for low values of $\beta_{G}<<1$. Dashed line is the value of enhancement (4) for $\alpha_{K L J}=0.56$

\section{Limits on the enhancement}

There are various reasons why unbounded enhancement doesn't occur. Even those aspects which were already discussed by Guduru and coworkers are given here in more details, in the new notation permitted by Kesari's equation, and new comparisons are added. 


\subsection{Deviations at large $\beta_{G}$}

As we have discussed in the previous paragraph, the Kesari expansion is valid in the limit $\widehat{a}>>1$, which implies in general low values of $\beta_{G}<<1$. As clear in the Fig.5 which compare the actual pull-off values from the full Guduru model with those of the asymptotic expansion, the enhancement is much reduced at large $\beta_{G}$. In particular, following the various curves in Fig.5 at increasing $\beta_{G}$, the enhancement is much reduced for intermediate values of $\alpha_{K L J}$ although the low $\alpha_{K L J}$ paradoxical behaviour seems preserved. Notice we can actually switch to reduction instead of enhancement for large $\beta_{G}$, but this is due, as noticed by Guduru (2007), to the fact that in the limit of very large wavelength (which is also large $\beta_{G}$ ), we have essentially only one contact at separation, that obtained the sphere with just the central crest of the wavy surface, having a reduced equivalent radius

$$
\frac{1}{R_{e f f}}=\frac{1}{R}+4 \pi^{2} \frac{A}{\lambda^{2}}
$$

If we consider the pull-off value with this reduced radius, this leads to

$$
\widehat{P}_{\mathrm{min}, \text { large } \beta_{G}}=-\frac{R_{e f f}}{R}=-\frac{1}{1+4 \pi^{2} \alpha_{G}}=-\frac{\pi^{3 / 2} \alpha_{K L J} \sqrt{\beta_{G}}}{\pi^{3 / 2} \alpha_{K L J} \sqrt{\beta_{G}}+4 \pi^{2}}
$$

which goes to 1 both for large $\beta_{G}$ and large $\alpha_{K L J}$ as it is clear from Fig.5.

Combinations of large $\beta_{G}$ and small $\alpha_{K L J}$ shows this reduction is not too large. Guduru suggested there is an optimal wavelength where the enhancement is highest; however, this must necessarily be just a different rewording of the dependences on the parameters. At low $\beta_{G}$, there is so far no real limit on the enhancement, if it wasn't for the conditions on the following paragraphs. 


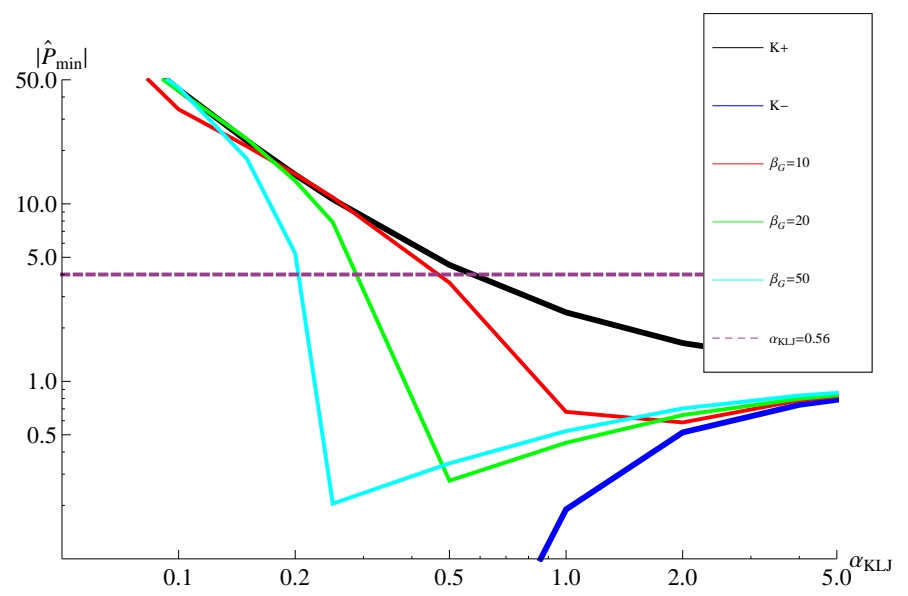

Fig.5 - Enhancement of pull-off. Black thick line is obtained with Kesari equation on unloading, blue line with Kesari equation on loading, and

red,green,cyan are obtained with the full Guduru solution with

$$
\beta_{G}=10,20,50
$$

\subsection{Contradiction of the simply connected area}

The enhancement predicted from the Kesari envelope curve on unloading becomes arbitrarily large at low $\alpha_{K L J}$ and indeed also Guduru (2007) in Fig.11, shows values of the order of 30-40 factor of increase, with values higher than 15 being actually measured in his experimental validation paper (Guduru \& Bull, 2007). Guduru (2007) has a very preliminary discussion about the validity of the assumption of simply connected contact area, as for the case of non-adhesive contact, it can be cast very clearly in terms of the parameter $\alpha_{G}=\frac{A R}{\lambda^{2}}$. In particular, to have the gap function monotonically increasing, it is enough to have

$$
\alpha_{G}=\frac{A R}{\lambda^{2}}=\frac{1}{\pi^{3 / 2} \alpha_{K L J} \sqrt{\beta_{G}}}<\frac{1}{8.5761}=0.12
$$

or

$$
\beta_{G}>\beta_{\lim }=\left(\frac{1.54}{\alpha_{K L J}}\right)^{2}
$$

Hence, at low $\alpha_{K L J}$ enhancement in the Guduru-Kesari model can only hold if $\beta_{G}$ is large, as otherwise separation in the contact may well occur. As we are interested in the range $\alpha_{K L J}<0.56$, the restriction on the shape requires $\beta_{G}>7.6$ or larger: however, this conflicts with the approximation 
in Kesari's envelope, so that in fact the enhancement should be computed from the full Guduru's equation, and the actual minimum of the curve is now much less than what expected from the envelope. An estimate of this is seen from Fig.5 of Waters et al (2009), where it is clear that for $\alpha_{G}<0.12$ we are generally below $\left|\widehat{P}_{\text {min }}\right|=4$. This is because at large $\beta_{G}$, we are looking at the problem of a single asperity detaching, as we have seen in point 1) of the discussion above, which reduced adhesion, instead of enhancing it.

In Fig.6, we plot the boundary defined by $\beta_{G}=\beta_{\text {lim }}$ with a red curve: notice that this corresponds to increasingly high values of $\beta_{G}$ the more we move towards the left. Only points below this curve are "certainly" satisfying the monotonicity of the punch and hence of the simply connected contact. It is clear that within this region, the enhancement is lower than 4 which is also the highest amplification with Johnson parameter $\alpha_{K L J}>0.56$.

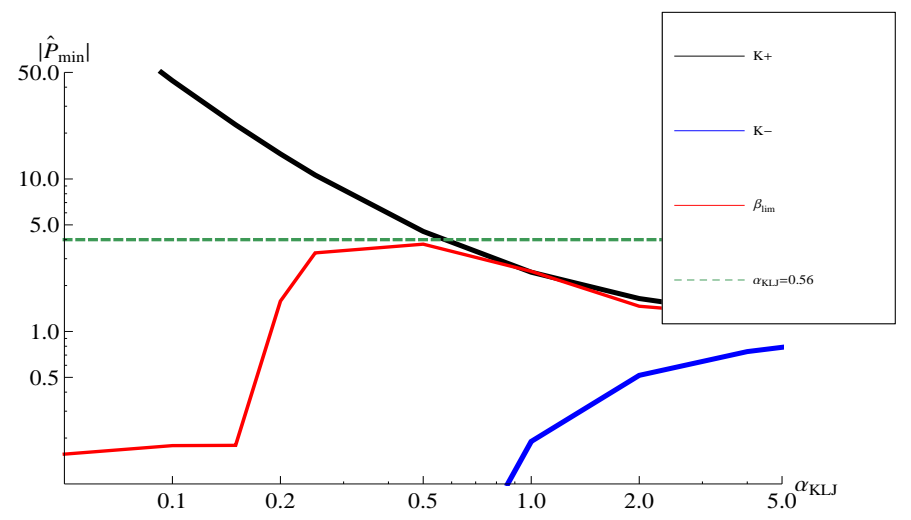

Fig.6 - The boundary of enhancement of pull-off. Black thick line is obtained with Kesari equation on unloading, in blue curve Kesari equation on loading, and red curve is $\beta_{G}=\beta_{\lim }\left(\alpha_{K L J}\right)$

Guduru (2007) notices that the condition $\alpha_{G}<0.12$ is too restrictive on two grounds: one, because of the effect of adhesion, which can only make the likelihood of contact greater: indeed, even from the single sinusoid solution that we described in the first paragraph, we know that the pressure to reach full contact decreases from the purely mechanical one $p^{*}=\pi E^{*} A / \lambda$ and could be defined as $p^{*}\left(\alpha_{K L J}\right)$, see Afferrante et al (2015). This is important as we know from that analysis, that once in full contact, there is little chance to return to separated contact (Johnson (1995) has to postulate a flaw at the interface, or else there is theoretical strength as the only possible limit), 
other than from the contact edge. Second, because the monotonicity of the punch profile is reobtained for large compressions, i.e. loading the contact substantially before starting the unloading. In particular, from the function of the profile, the condition is immediately found as

$$
\frac{1}{4 \pi^{2} \alpha_{G}}>-\frac{\sin (2 \pi r / \lambda)}{(2 \pi r / \lambda)}
$$

which has a maximum giving the absolute $\alpha_{G}<0.1166$, but otherwise should be satisfied for $r>r_{c}$, where one choice is

$$
\frac{r_{c}}{\lambda}=2 \pi A \frac{R}{\lambda^{2}}=2 \pi \alpha_{G}>>2 \pi \cdot 0.12=0.75
$$

and indeed $1>-\sin \left(4 \pi^{2} A \frac{R}{\lambda^{2}}\right)$. This is not further discussed in Guduru (2007), but here we will make more considerations. First of all, we should notice that to have the radius of contact less than, say, $1 / 4$ of the radius of the sphere, we need $2 \pi \frac{A}{\lambda} R<\frac{R}{4}$, which gives

$$
\frac{A}{\lambda}<\frac{1}{8 \pi}=0.04
$$

This together with $\alpha_{G}=\frac{A R}{\lambda^{2}}>0.12$ gives $\frac{R}{\lambda}>\frac{0.12}{A / \lambda}=3$ or higher. With respect to $\alpha_{K L J}$, the condition on $\frac{A}{\lambda}<\frac{1}{8 \pi}$ leads to $\alpha_{K L J}>4 \sqrt{\frac{l_{a}}{\pi A}}$, where $l_{a}=w / E^{*}$. Since we want $\alpha_{K L J}<0.56$, for example $\alpha_{K L J}=0.1$, then we need $\frac{l_{a}}{A}<\pi\left(\frac{0.1}{4}\right)^{2}$ or $A>510 l_{a}$. But $A<\frac{\lambda}{8 \pi}$ so that $\lambda>12818 l_{a}$, and therefore $R>38454 l_{a}$, so we need big enough spheres with respect to adhesion characteristic length.

Let us further estimate the pressure distribution (from Guduru (2007), under adhesionless contact only) with respect to $p^{*}=\pi E^{*} A / \lambda$, noting that with $\frac{A}{\lambda}=0.04$ this corresponds to a quite high compressive stress, just $1 / 10$ of the elastic modulus, with the warning that finite strains and other deviations may occur. We could convince ourselves that the waviness has been squashed out mechanically if the local pressure is positive. Now, rearranging Guduru's equation in the form

$$
\frac{p}{p^{*}}\left(\frac{r}{a}\right)=2\left(\frac{1}{\pi^{2} \alpha_{G}}+2\right) \frac{a}{\lambda} \sqrt{1-\left(\frac{r}{a}\right)^{2}}+\int_{r / a}^{1} \frac{H_{0}\left(2 \pi \frac{x}{a} \frac{a}{\lambda}\right)}{\sqrt{\left(\frac{x}{a}\right)^{2}-\left(\frac{r}{a}\right)^{2}}} d \frac{x}{a}-2 \pi\left(\frac{a}{\lambda}\right) \int_{r / a}^{1} \frac{\frac{x}{a} H_{1}\left(2 \pi \frac{x}{a} \frac{a}{\lambda}\right)}{\sqrt{\left(\frac{x}{a}\right)^{2}-\left(\frac{r}{a}\right)^{2}}} d \frac{x}{a}
$$


we can plot it for $\frac{a}{\lambda}=\frac{r_{c}}{\lambda}=2 \pi \alpha_{G}$ (Fig.7a) and $\alpha_{G}=0.05,0.1,1,5$, as well as for even higher contact radius $\frac{a}{\lambda}=3 \frac{r_{c}}{\lambda}=6 \pi \alpha_{G}$. (Fig.7b). The results show that at the very low $\alpha_{G}=0.075$ (thick black line) the pressure is indeed always compressive and this should occur for any value of the contact area. However, some tension appears for $\alpha_{G}=0.15$ (thick blue line) or higher values, which is where the "local" monotonicity condition postulated by Guduru (2007) should also suggest compression always for any value of the contact area. Only with triple radius of contact, as in Fig.7b, we do find always compressive stresses. In the case of adhesion, since we are interested in the case $\alpha_{K L J}<0.56$, there is no guarantee therefore that the "local" condition of Guduru (2007) justifies the simple connected area assumption. This, together with the fact that the pressure may be impractically large, suggests that we may not be able to observe the actual enhancement predicted by the simply connected contact area solution. 


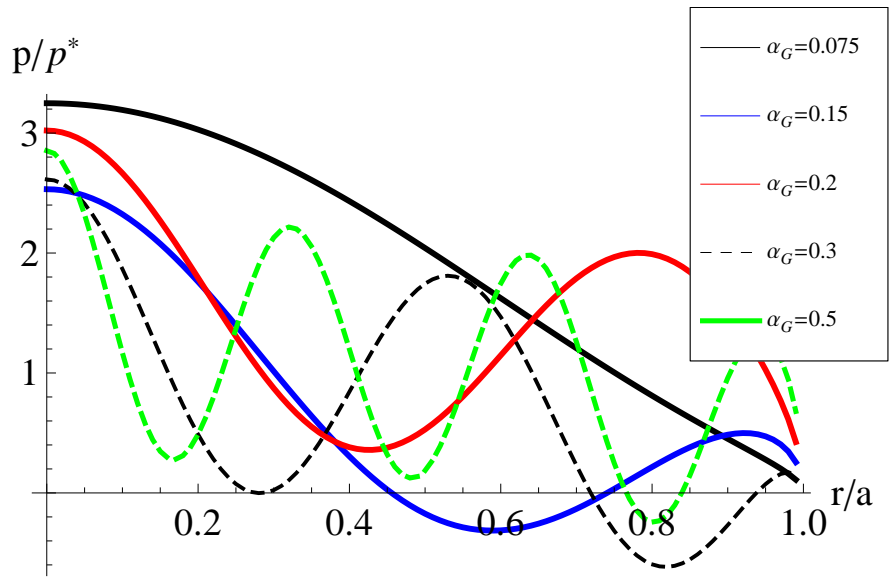

(a)

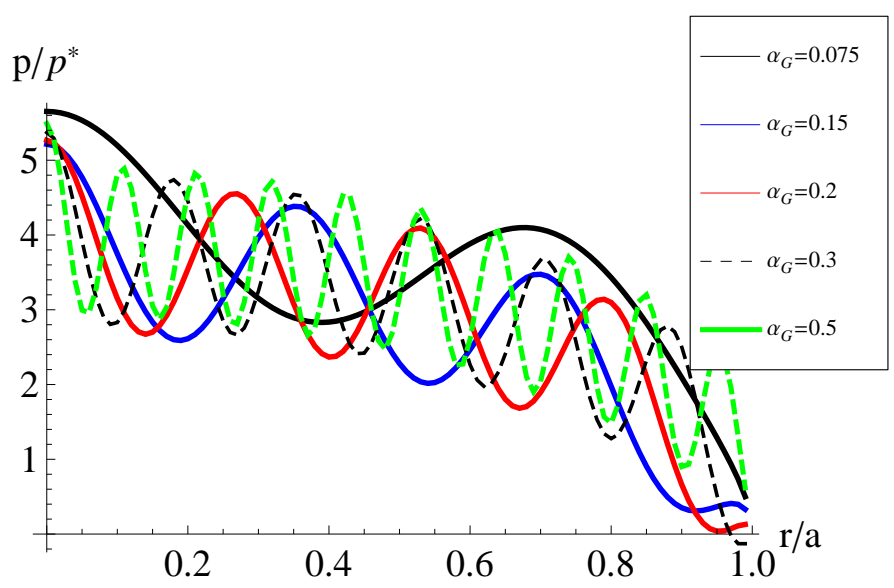

Fig.7 - Pressure distributions with pure mechanical contact, for $\alpha_{G}=0.075,0.15,0.2,0.3,0.5$ and for contact radius (a) $\frac{a}{\lambda}=\frac{r_{c}}{\lambda}=2 \pi \alpha_{G}$ or (b) $\frac{a}{\lambda}=3 \frac{r_{c}}{\lambda}=3 \times 2 \pi \alpha_{G}$

\section{Deviation from the JKR regime}

The transition in adhesion from Bradley-rigid behavioun 3 to fully elastic JKR behaviour is well known for the sphere. It depends on the well known

\footnotetext{
${ }^{3}$ It is more precise to say from rigid behaviour to JKR regime, as the DMT solution, commonly referred to as the limit for low Tabor parameter, has indeed various forms, and most of them not exact.
} 
Tabor parameter

$$
\mu=\frac{\sigma_{t h}}{E^{*}}\left(\frac{9 R E^{*}}{2 \pi w}\right)^{1 / 3}
$$

where $\sigma_{t h}$ is the theoretical strength and we have defined the parameter with reference to the sphere, but we could also define a value appropriate for the roughness.

Anyway, the phenomenon of enhancement of toughness and strength because of surface waviness has been shown to be restricted primarily to the JKR adhesion regime in Waters et al (2009), or $\mu>1$. This is important in view of application of the Guduru model to rough surfaces. Guduru and Kesari have considered very soft materials with very small amounts of roughness. If we consider the more standard situation of macroscopic contacts with many asperities, the local behaviour of asperities will not show any enhancement. However, in intermediate situations the situation is unclear.

However, a further remark is that the limit of "rigid" roughness is not necessarily that of the sphere without roughness, as it seems suggested in Waters et al (2009). Indeed, first of all, we have the limit at large $\beta_{G}$ which should reduce the pull-off to the case of contact between the sphere and the first crest of the waviness. Secondly, we have at our disposal a solution for rigid adhesion between a spherical particle and a rough plane (simplified with a single small asperity, and otherwise smooth plane), due to Rumpf (1990), later modified by Rabinovich et al (2000) which is obtained applying Derjaguin's approximation and contains two terms: a first term represents the interaction sphere/asperity (which increases with $r$ ), and "noncontact" particle/flat separated by the height (radius) of the asperity (decreasing with $r)$

$$
\frac{P_{a d}}{P_{\text {smooth }}}=\frac{1}{1+R / r}+\frac{1}{\left(1+r / H_{0}\right)^{2}}
$$

where $H_{0}$ is some atomic size length scale. This equation has been used very much in the area of particle adhesion and powder technology, as well as drug delivery, semiconductor fabrication, xerographic processes, and paint formulation or aerosol formation, amongst others) and shown to be reasonably in

\footnotetext{
${ }^{4}$ Rabinovicz et al (2000) modified this to take into account a one-scale roughness but this simply changes the radius of the asperity into $r=1.485 \mathrm{rms}$, but the behaviour is the same. In the nanoscale roughness regime, a dramatic decrease in adhesion force is predicted for this size adhering particle.
} 
agreement with experiments particularly at nanoscale - despite its simplicity and the strong assumption of negligible elastic deformations.

This Rumpf-Rabinovich model is very useful for our purpose as, for both extremely small "roughness" (subatomic roughness) $r / R \rightarrow 0$, or very large radius of asperity $r$ (when the contact is essentially between the sphere and a single asperity which in this case has grown in curvature so that it is a flat plane itself), this model leads to the value for the particle alone on the flat surface $P_{\text {smooth }}$ which is in this case the Bradley result for the sphere, $2 \pi R w$. This is the value Waters et al (2009) seem to obtain for $\mu \rightarrow 0$.The model shows therefore with increasing $r / R$ first a decrease of adhesion due to roughness and then, after reaching a minimum, an increase towards a linear trend in $\mathrm{R}$ of the Bradley equation.

$$
\begin{aligned}
& \text { In Guduru's notation } r=\frac{\lambda^{2}}{A(2 \pi)^{2}} \text { and } \\
& \qquad \frac{r}{R}=\frac{1}{(2 \pi)^{2} \alpha_{G}}
\end{aligned}
$$

and therefore the Rumpf-Rabinovich model gives

$$
\frac{P_{a d}}{P_{\text {smooth }}}=\frac{1}{1+(2 \pi)^{2} \alpha_{G}}+\frac{1}{\left(1+\frac{\lambda^{2}}{A H_{0}(2 \pi)^{2}}\right)^{2}}
$$

This formula has been shown to work pretty well with nano and atomic size roughness, with hard solids. In our case, as we are uncertain especially of the case $\alpha_{G}>0.12$, corresponding to $\frac{r}{R}<0.21$, it is clear that the first term is small (interaction sphere asperity), of 0.174 and less. On the other hand, the second term giving the interaction with the smooth plane separated by the hemiasperity is also extremely small too when $r>>H_{0}$ (atomic size) as it is common. Suppose $R=10^{9} H_{0}$, and the Rumpf-Rabinovich model gives

$$
\frac{P_{a d}}{P_{\text {smooth }}}=\frac{1}{1+(2 \pi)^{2} \alpha_{G}}+\frac{1}{\left(1+\frac{10^{9}}{(2 \pi)^{2} \alpha_{G}}\right)^{2}}
$$

We explore the range $r / H_{0}=1,10^{9}$, i.e. $\alpha_{G}=\frac{1}{(2 \pi)^{2}}, \frac{10^{9}}{(2 \pi)^{2}}$ in Fig.8 below, which clearly shows that reduction of pull-off of various orders of magnitude for $\alpha_{G}$ increasing in the range where we cannot assume full contact. 


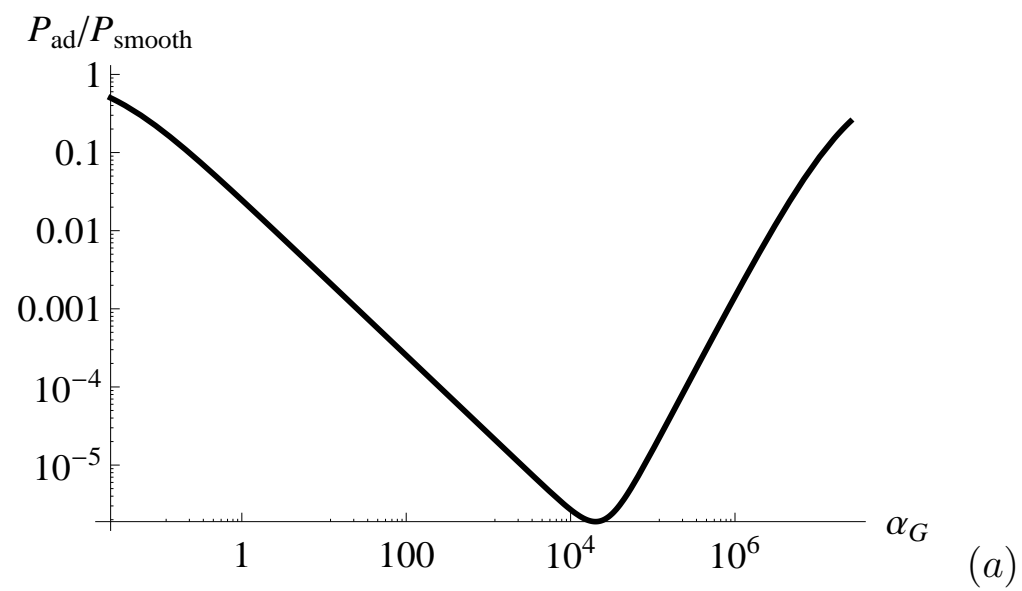

Fig.8 - The Rumpf-Rabinovich model as applied to the Guduru problem

It is clear that, by assuming a simply connected contact area, Waters et al (2009) found only a very limited range of possible decay of adhesion, although they should have found at least the regime at high $\beta_{G}$ where the contact is expected between the sphere and the first crest of the sinusoidal waviness, therefore reduced with respect to the Bradley result for the sphere.

\section{Conclusions}

We have revisited the Guduru model, and discussed the possible reasons for the limitation of the very high enhancement of adhesion found in that model. In particular, we have observed a reduced dependence on the parameters, we have identified the Johnson parameter for single sinusoidal contact to govern also the amplification of adhesion, at least in an asymptotic regime where the envelope solution by Kesari et al holds.

Finally, we have shown that, as very large amplification is expected from the Guduru model, even greater reduction is expected in the separated contact regime, as estimated from a rigid model adhesion equation by Rumpf and Rabinovich. The latter gives perhaps the lower bound of the adhesion, as Guduru gives the upper bound.

\section{References}

Afferrante, L., Ciavarella, M., \& Demelio, G. (2015). Adhesive contact of the Weierstrass profile. In Proc. R. Soc. A (Vol. 471, No. 2182, p. 20150248). The Royal Society. 
Briggs G A D and Briscoe B J (1977) The effect of surface topography on the adhesion of elastic solids J. Phys. D: Appl. Phys. 10 2453-2466

Ciavarella, M., Demelio, G., Barber, J. R., \& Jang, Y. H. (2000). Linear elastic contact of the Weierstrass profile. In Proceedings of the Royal Society of London A: Mathematical, Physical and Engineering Sciences (Vol. 456, No. 1994, pp. 387-405). The Royal Society.

Ciavarella, M. (2016). Adhesive rough contacts near complete contact. in press, Int J Mech Sci., arXiv preprint arXiv:1504.08240.

Fuller, K. N. G., \& Tabor, D. (1975). The effect of surface roughness on the adhesion of elastic solids. Proc Roy Soc London A: 345, No. 1642, 327-342

Fuller, K.N.G. , Roberts A.D. (1981). Rubber rolling on rough surfaces J. Phys. D Appl. Phys., 14, pp. 221-239

Gao, H., \& Yao, H. (2004). Shape insensitive optimal adhesion of nanoscale fibrillar structures. Proceedings of the National Academy of Sciences of the United States of America, 101(21), 7851-7856.

Greenwood, J. A. (2007). On the DMT theory. Tribology Letters, 26(3), 203-211.

Guduru, P.R. (2007). Detachment of a rigid solid from an elastic wavy surface: theory J. Mech. Phys. Solids, 55, 473-488

Guduru, P.R. , Bull, C. (2007). Detachment of a rigid solid from an elastic wavy surface: experiments J. Mech. Phys. Solids, 55, 473-488

Hui, C. Y., Glassmaker, N. J., Tang, T., \& Jagota, A. (2004). Design of biomimetic fibrillar interfaces: 2. Mechanics of enhanced adhesion. Journal of The Royal Society Interface, 1(1), 35-48.

Johnson, K. L., K. Kendall, and A. D. Roberts. (1971). Surface energy and the contact of elastic solids. Proc Royal Soc London A: 324. 1558.

Kesari, H., Doll, J. C., Pruitt, B. L., Cai, W., \& Lew, A. J. (2010). Role of surface roughness in hysteresis during adhesive elastic contact. Philosophical Magazine \& Philosophical Magazine Letters, 90(12), 891-902.

Kesari, H., \& Lew, A. J. (2011). Effective macroscopic adhesive contact behavior induced by small surface roughness. Journal of the Mechanics and Physics of Solids, 59(12), 2488-2510.

Kamperman, M., Kroner, E., del Campo, A., McMeeking, R. M., \& Arzt, E. (2010). Functional adhesive surfaces with "gecko" effect: The concept of contact splitting. Advanced Engineering Materials, 12(5), 335-348. 
Persson, B.N.J. (2002). Adhesion between an elastic body and a randomly rough hard surface, Eur. Phys. J. E 8, 385-401

Rabinovich, Y. I., Adler, J. J., Ata, A., Singh, R. K., \& Moudgil, B. M. (2000). Adhesion between nanoscale rough surfaces: I. Role of asperity geometry. Journal of Colloid and Interface Science, 232(1), 10-16.

Rumpf, H. Particle Technology, Chapman \& Hall, London/New York (1990)

Yao, H., \& Gao, H. (2006). Mechanics of robust and releasable adhesion in biology: Bottom-up designed hierarchical structures of gecko. Journal of the Mechanics and Physics of Solids, 54(6), 1120-1146.

Waters, J.F. Leeb, S. Guduru, P.R. (2009). Mechanics of axisymmetric wavy surface adhesion: JKR-DMT transition solution, Int J of Solids and Struct 46 5, 1033-1042 